\title{
ON A PROPERTY OF FUNCTIONS WITH NON-NEGATIVE DERIVATIVES AT THE ORIGIN AND ITS APPLICATION ${ }^{1}$
}

\author{
R. G. LAHA
}

Communicated by J. L. Doob, October 7, 1960

Functions which have non-negative derivatives at the origin play an important role in the theory of probability. The moment generating functions of symmetric distributions are the most common examples of such functions. In the present note we first give a theorem on the properties of such functions. Finally we consider an interesting application of this theorem in deducing an analytic decomposition of the Poisson distribution. For this purpose we require the following definition:

Definition. Let $g(x)$ be a real-valued function of the real variable $x$ which has finite derivatives of all orders

$$
g^{(k)}(0)=\left[\frac{d^{k}}{d x^{k}} g(x)\right]_{x=0}, \quad k=1,2,3, \cdots,
$$

at the point $x=0$. We then define formally the function

$$
G(z)=g(0)+\sum_{k=1}^{\infty} \frac{g^{(k)}(0)}{k !} z^{k} \quad(z \text { complex })
$$

as a function of the complex variable $z(z=x+i y, x$ and $y$ being both real) and call $G(z)$ the adjoint function of $g(x)$. In general, the function $G(z)$ may not exist, except at the point $z=0$ and we note that $G(0)=g(0)$. The adjoint function $G(z)$ is said to be regular in the circle $|z|<R(R>0)$ about the point $z=0$ if and only if the power series $g(0)+\sum_{k=1}^{\infty}\left(g^{(k)}(0) / k !\right) z^{k}$ converges in the same circle $|z|<R$. We can now formulate the following theorem:

Theorem 1. Let $\left\{g_{n}(x)\right\}, n=1,2, \cdots$, be a countable sequence of real valued functions of the real variable $x$ such that $g_{n}(x) \geqq 1$ for $0<x<\delta$ $(\delta>0)$ and further each $g_{n}(x)$ has non-negative derivatives of all orders at the point $x=0$. Let $\left\{\alpha_{n}\right\}$ be a sequence of positive numbers such that $0<\alpha_{0} \leqq \alpha_{n} \leqq \alpha_{0}^{\prime}, n=1,2, \cdots$. Let $g(x)$ be a function of the real variable $x$ having finite derivatives of all orders at the point $x=0$ such that the corresponding adjoint function $G(z)$ is regular in a circle $|z|<R(R>0)$ about the point $z=0$ and has no zeros inside this circle. Suppose that the

1 This work was supported by the National Science Foundation through grant NSF-G-9968. 
infinite product $\prod_{n-1}^{\infty}\left\{g_{n}(x)\right\}^{\alpha_{n}}$ converges uniformly in the interval $0<x \leqq \delta(\delta>0)$ and satisfies the relation

$$
\prod_{n=1}^{\infty}\left\{g_{n}(x)\right\}^{\alpha_{n}}=g(x)
$$

for all real $x$ in the interval $0<x \leqq \delta(\delta>0)$.

Then each of the adjoint functions $G_{n}(z)$ corresponding to $g_{n}(x)$ is also regular at least in the circle $|z|<R$ and further the relation

$$
\prod_{n=1}^{\infty}\left\{G_{n}(z)\right\}^{\alpha_{n}}=G(z)
$$

holds for all complex $z$ in the circle $|z|<R$.

The method of proof of the theorem is similar to the one used by Mamay in [5] who has deduced an analogous theorem on the properties of positive definite functions. This work was stimulated by a question raised by Linnik in [4]. The special case of this theorem when the number of factors is finite was already proven by Dugué [2]. An alternative proof has been given by the author in [3].

The following analytical decomposition of the Poisson distribution has been obtained as an application of this theorem:

THEOREM 2. Let $\left\{f_{n}(t)\right\}$ be a countable sequence of characteristic functions $^{2}$ and let $\left\{\alpha_{n}\right\}$ be a sequence of positive numbers such that $0<\alpha_{0}$ $<\alpha_{n}<\alpha_{0}^{\prime}$. Suppose that the functions $f_{n}(t)$ satisfy the relation

$$
\prod\left\{f_{n}(t)\right\}^{\alpha_{n}}=\exp \left\{\lambda\left(e^{i t}-1\right)\right\}(\lambda>0)
$$

for all real $t$ in a certain neighborhood $|t|<\delta(\delta>0)$ about the point $t=0$. Then each factor $f_{n}(t)$ has the form

$$
f_{n}(t)=\exp \left\{i t \mu_{n}+\lambda_{n}\left(e^{i t}-1\right)\right\}
$$

where $\lambda_{n} \geqq 0$ and $\mu_{n}$ are real numbers.

This theorem, for the case of a finite number of factors, has been proved by Dugué $[1 ; 2]$ which may be considered as an extension of the theorem of Raikov [6] in an analytical direction.

\section{REFERENCES}

1. D. Dugue, Resultats sur les fonctions absolument monotones et applications d l'arithmetique de fonctions de type positif, C.R. Acad. Sci. Paris. vol. 244 (1957) pp. 715-717.

A characteristic function is the Fourier-Stieltjes transform of a distribution function. 
2. Sur le theorème de Levy-Cramer, Publ. Inst. Statist. Univ. Paris vol. 6 (1957) pp. 213-225.

3. R. G. Laha, On functions having non-negative derivatives at the origin, Proc. Amer. Math. Soc., to appear.

4. Yu. V. Linnik, A problem on characteristic functions of probability distributions, (Russian) Uspehi. Mat. Nauk. vol. 10 (1955) pp. 137-138.

5. L. V. Mamay, On the theory of characteristic functions, (Russian) Vestnik Leningrad Univ. vol. 15 (1960) pp. 85-99.

6. D. A. Raikov, On the decomposition of Gauss and Poisson laws, (Russian) Izv. Akad. Nauk SSSR. Ser. Mat. vol. 2 (1938) pp. 91-124.

The Catholic University of America

\title{
SUMMABILITY $(L)$ OF FOURIER SERIES
}

\author{
FU CHENG HSIANG
}

Communicated by S. Bochner, September 28, 1960

1. In a recent paper, Borwein [1] has constructed a new method of summability for an infinite sequence $\left(S_{n}\right)$. He defined a sequence $\left(S_{n}\right)$ to be summable by the logarithmic method of summability or summable $(L)$ to the sum $s$ if, for $x$ in the interval $(0,1)$

$$
\lim _{x \rightarrow 1-0} \frac{1}{|\log (1-x)|} \sum_{n=1}^{\infty} \frac{S_{n}}{n} x^{n}=s,
$$

which is written simply as $S_{n} \rightarrow s(L)$. Concerning this kind of summability, Borwein has established a number of fundamental facts. For instance, he showed that $(L) \supset(A, \lambda) .{ }^{1}$ Thus, we have the following full inclusive relation:

$$
(L) \supset(A, \lambda) \supseteq(A) \supset(C, r),
$$

for any $r>-1$, where $(A)$ is the ordinary Abel's summability and $(C, r)$ is the Cesàro summability of order $r$.

In this note, the author intends to apply this new method of summability to the Fourier series of $f(x)$ in order to obtain a corresponding summability criterion for it.

1 A sequence $\left(S_{n}\right)$ is said to be summable $(A, \lambda)$ to the sum $s$ if $(1-x) \sum S_{n}^{\lambda} x_{n} \rightarrow s$ as $x \rightarrow 1-0$, where $S_{n}^{\lambda}$ is the $n$th Cesaro mean of order $\lambda$ of $\left(S_{n}\right)[1$, p. 212 and $\delta 3$, Theorem 3]. 